\title{
Simulation Method for Scheduling Linear Construction Projects Using the Learning- Forgetting Effect
}

\author{
Łukasz Rzepecki ${ }^{1, *}$, and Stawomir Biruk ${ }^{1}$ \\ ${ }^{1}$ Lublin University of Technology, Faculty of Civil Engineering and Architecture, \\ Nadbystrzycka 38D, 20-618 Lublin, Poland
}

\begin{abstract}
Linear projects such as highways, multi-storey buildings or pipelines are implemented by repeating the sequences of processes on units. Repetitive operations affect acquiring more and more experience, which shortens the duration of subsequent activities. On the other hand, the possible breaks in work make this effect disappear. Breaks at work are most often caused by the lack of crew work continuity. While delaying the subsequent sequences of processes properly, it is possible to reduce interruptions and limit the forgetting effect. A simulation method for scheduling linear construction projects has been developed, taking into account the learning and forgetting effect. It allows to increase the probability of the crew work continuity while meeting the project deadline with a specific probability. As a result, the estimation of the project duration is more accurate, which may provide an advantage at the stage of submitting offers in tenders for construction works.
\end{abstract}

\section{Introduction}

Construction projects encompass within their scope frequently repeated works conducted on identical or similar work units. Such projects are exemplified by multi-storey buildings, highways and pipelines. Identically composed crews carry out construction processes on successive work units in the same cyclical and parallel order, with each subsequent process beginning only after work on a given unit has been completed by the previous crew.

The Critical Path Method (CPM) is not effective for scheduling repetitive processes. Methods based on the Line of Balance (LOB) concept are usually applied to plan linear projects. These methods focus on scheduling tasks for carefully selected work crews by coordinating activities in time in such a way as to eliminate unnecessary idle periods in the operation of resources. The most well-known methods deriving from LOB include Linear Scheduling Method (LSM) [1], Productivity Scheduling Method (PSM) [2-3], Vertical Production Method (VPM) [4] and Repetitive Scheduling Method (RSM) [5]. They apply graphic modelling, optimization methods, elements of network planning and, most importantly, take into account constraints between activities executed on the same floor and a different floor [6]. All these planning techniques fall under the umbrella term of Linear

${ }^{*}$ Corresponding author: 1.rzepecki@,pollub.pl 
Scheduling Method (LSM). The analysis of interconnections between processes executed on successive work units allows establishing the controlling sequence of processes, whose practical importance is the same as that of the critical path for CPM [5].

The majority of existing methods for scheduling construction projects is applicable under deterministic conditions. While schedules developed this way greatly facilitate modelling complex projects, they are prone to fall out of date under real-life conditions. This is true particularly of the building industry which differs largely from manufacturing where production takes place under stable technical and organizational conditions. Construction is more exposed to risk factors and uncertainty than other branches of national economy [7]. The effect of external factors on the implementation of a building project is magnified by a large number of participants and long implementation cycles. Complexity of an undertaking and the period of its implementation increase in step with the force of risk factors, as it becomes more difficult to evaluate their probability and predict their possible effects [8]. A schedule should be developed for an acceptable risk level. A commonly used way to make schedules more robust against disruptions is to allocate time buffers (reserves). Time buffers are used to ensure the timely completion of individual processes and the project as a whole. The main problem is to size the time buffers adequately, as this may affect the duration of the works.

\section{The learning-forgetting effect}

As the same work processes are repeated in successive units, work crews acquire experience which helps to shorten lead times and reduce labour costs. Developing a schedule requires determining work efficiency to allow tracking the time and cost of the undertaking. In the case of projects consisting of repetitive processes, the scheduling stage should allow for past experience contributing to reducing the work time, in order to gain an edge at the bidding stage.

The learning theory aims to mathematically describe the relations between work repetition and efficiency. This is true where workers carry out standard productive operations, whereby they improve their skills and possibly also learn how to use their workplace in a more ergonomic way.

The oldest basic learning model is Wright's exponential model [9] from 1936:

$$
t_{n}=t_{1} \cdot n^{-l}
$$

where:

$t_{n} \quad$ lead time of process performed on work unit $n$,

$t_{1} \quad$ lead time of process performed on the first work unit,

$n \quad$ number of process repetitions,

$l$ reduction parameter reflecting the slope of logarithmic curve; it is assumed that $l=-\frac{\log s}{\log 2}$, and

$s \quad$ known as learning rate - is defined as a time reduction percentage that results in doubling the production output.

Work interruptions mean that crews need longer times to complete operations on successive units. Short interruptions lead to a partial loss of experience, while in the case of sufficiently long ones, the loss may be complete [10]. Forgetting as a result of work interruptions is a reverse process to acquiring experience $[11,12]$ and contributes to longer lead times. Globerson and Levin [11] proposed the so-called S-shaped Forgetting Function, 
which is easily applicable to the simulation method for the reason that the forgetting effect depends directly on the length of work interruption $\Delta t$ :

$$
t_{b}=t_{1}-\left[t_{1}-t_{w}\right] \cdot(a \cdot \Delta t+1) \cdot e^{-a \cdot t},
$$

where:

$t_{w} \quad$ duration of subsequent process if no interruption occurred,

$a$ forgetting coefficient,

$e \quad$ base of natural logarithm.

The effect of experience on work efficiency was also surveyed in the construction industry, for example, in works related to reinforced concrete floors [13], roof coating [14], reinforced concrete piles [15] or installing rebars [16]. The learning curve was also used for planning motorway construction [17] as well as the installation of underwater caissons [18]. It is usually assumed that the rate of experience for most processes within the construction industry ranges from $70 \%$ to $95 \%$.

The learning-forgetting theory was also used for scheduling construction projects. Lam et al. [10] used Wright's model [9] and S-shaped Forgetting Function [11] to plan undertakings by the LOB method under deterministic conditions. Lutz et al. [19] took into account the learning effect only in a repetitive process simulation. They assumed that the duration times of construction processes, might be shortened by up to $25 \%$ due to the experience curve.

\section{The method of determining time buffers for linear construction project planning}

A sequence of processes $i(i=1,2, \ldots, n)$ is to be performed on each unit $j(j=1,2, \ldots, m)$ in unchanged order. The time for completion of works by crew $i$ on unit $j$ equals $t_{i j}$. The starting date of the first process on the first unit is $t_{11}^{s}=0$ (Fig. 1).

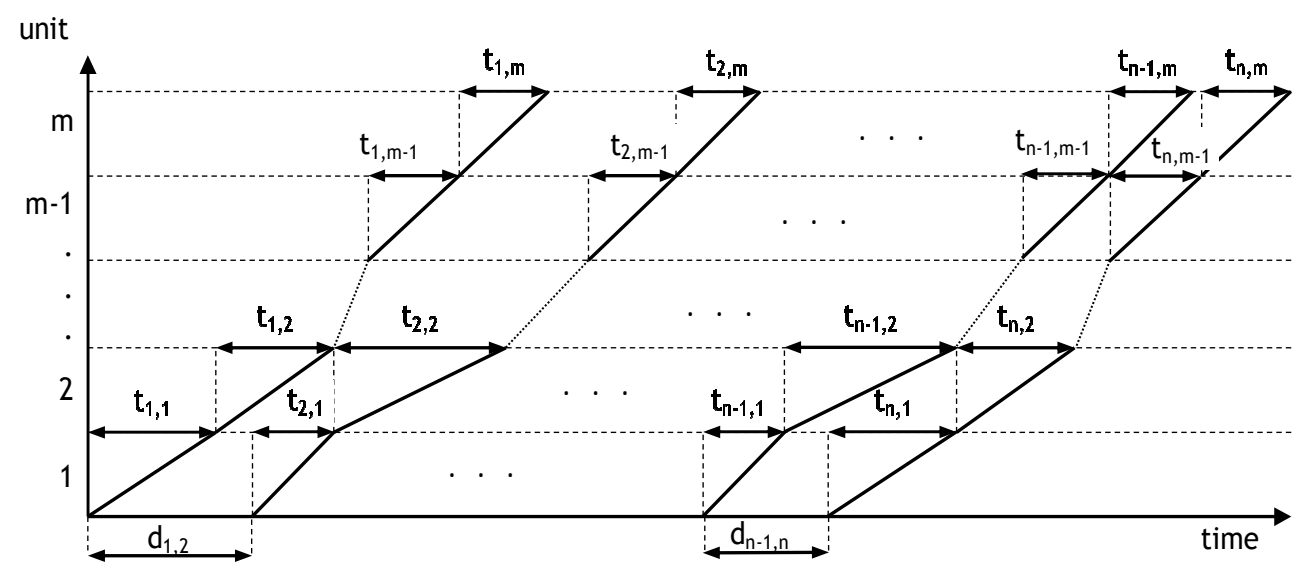

Fig. 1. Line of Balance diagram.

The shortest project cycle with crews operating continuously can be achieved at critical (maximum) proximity of the neighbouring broken lines (sections on the Line of Balance diagram) reflecting the progress of individual processes. The process $i+1$ should start relative to $i$ with a delay of $d_{i},(i+1)$. This delay can be calculated under deterministic conditions using the formula below: 


$$
d_{i,(i+1)}=\max \left\{\begin{array}{l}
t_{i 1} \\
t_{i 1}+t_{i 2}-t_{(i+1) 1} \\
\ldots \\
\sum_{j=1}^{m} t_{i j}-\sum_{j=1}^{m-1} t_{(i+1) j}
\end{array},\right.
$$

The following formula defines finish dates for processes within the first series string on successive work units $j(j=1,2, \ldots, m)$ and start dates for subsequent processes $(j+1)$ :

$$
t_{1 j}^{f}=\sum_{k=1}^{j} t_{1 k}
$$

while the start date for the subsequent process $(i \geq 2)$ can be defined using another formula, namely:

$$
t_{i j}^{s}=\sum_{s=1}^{i} d_{(s-1), s}+\sum_{k=1}^{j-1} t_{i k}
$$

Under random conditions, lead times $t_{i j}$ for processes $i(i=1,2)$ on successive units $j(j=1,2, \ldots, m)$ are random variables $T_{i j}$ with expected (average) value $E\left(T_{i j}\right)$ and variance $D^{2}\left(T_{i j}\right)$. Random variable parameters can be established by analogy to PERT for the following variants: $t_{i j}^{a}$ - optimistic time estimate, $t_{i j}^{m}$ - most likely time estimate, and $t_{i j}^{b}-$ pessimistic time estimate. Random variables exhibit the effect that the process finish and start dates scheduled according to formulas (4) - (5) fall out of date, leading to a possible discontinuity in crew operations and extending the lead time for the entire project.

The following procedure is proposed as a way for repetitive-process project schedules to increase the probability of work continuity, while also ensuring that the crews can meet the baseline finish date with a specific probability.

1. Define parameters types for the time distribution of the duration of construction processes. The formula below should be used to define the distribution function of project duration by the Monte Carlo method, without taking into account the learning effect but considering that the processes begin at the earliest possible dates (directly after the completion of a previous process on the same unit and the completion of works on the prior unit):

$$
t_{i j}^{s}=\max \left\{\begin{array}{c}
t_{(i-1), j}^{s}+t_{(i-1), j} \\
t_{i,(j-1)}^{s}+t_{i,(j-1)}
\end{array},\right.
$$

where:

$t_{i j} \quad$ crew work time $i(i=1,2, \ldots, n)$ on unit $j(j=1,2, \ldots, m)$ and $t_{11}^{s}=0$.

2. Agree with investor on baseline finish date $T_{d}$ and assess probability $R$ of meeting that time-limit based on a previously calculated distribution function.

3. Perform model simulation with regard to the learning-forgetting effect and assuming that processes start at earliest possible dates. The learning rate and forgetting coefficient should be based on the contractor's real-life expertise or literature describing relevant tests performed under comparable conditions. Calculate total idle time for all crews. The baseline finish date $T_{d}$ will be met with probability $R$ due to process duration shortened 
as a result of learning. This allows for a time reserve that can be used to increase the work continuity without altering the probability of timely completion on baseline finish date.

4. Delay start date of works (process strings performed by the same crew) and improve solution - introduce Buffer Times $d_{i,(i+1)}$. Delaying the start date of works contributes to reducing idle times, whereby forgetting effect is eliminated. Minimalizing of forgetting effect causes, as a result, shortens process duration times. Buffer Times may not affect the project's baseline finish date. This stage analyses project variants for different values of $d_{i,(i+1)}$, where process start date $i j$ is the greater of the values calculated on the basis of (5) and (6). The acceptable variant is a solution which meets the baseline finish date. The total crew idle time is calculated for each variant. This test aims to find the minimum idle time variant. This paper seeks to find a suboptimal solution through a heuristic algorithm simple hill climb algorithm. In the next step, an optimization algorithm is developed and implemented in simulation software.

\section{The learning-forgetting theory applied to schedule crew works related to the implementation of repetitive construction processes under random conditions}

This section presents the proposed method used as an example to schedule interior finish works in a multi-storey building. It is assumed that each floor requires the same amount of work, while crew efficiency depends on equipment efficiency (impossible to modify continuously duration time of processes), leading to variation in lead times for successive construction processes conducted on the same work unit. Each floor requires five construction processes in succession. The following triangular time distributions for work duration have been adopted here (Table 1).

Table 1. Durations of construction processes conducted on each floor.

\begin{tabular}{|c|c|c|c|}
\hline Process & $\begin{array}{c}\text { Optimistic time } \\
\text { estimate } \boldsymbol{t}_{\boldsymbol{a}} \\
\text { [days] }\end{array}$ & $\begin{array}{c}\text { Most likely time } \\
\text { estimate } \boldsymbol{t}_{\boldsymbol{m}} \\
\text { [days] }\end{array}$ & $\begin{array}{c}\text { Pessimistic time } \\
\text { estimate } \boldsymbol{t}_{\boldsymbol{b}} \\
\text { [days] }\end{array}$ \\
\hline Partition walls & 30 & 31 & 35 \\
\hline Plasters & 13 & 15 & 19 \\
\hline Backings & 36 & 38 & 43 \\
\hline Painting & 17 & 19 & 22 \\
\hline Floors & 14 & 15 & 17 \\
\hline
\end{tabular}

The simulation model implements Wright's exponential learning model - formula (1) and Globerson and Levin's forgetting model - formula (2).

It is assumed that the learning rate and forgetting coefficient are the same for each process, standing at $s=0.95$ and $a=0.15$, respectively. Independent random number generators (in the range from 0 to 1 ) were used to produce random variables representing the duration of construction work. The simulator was developed in GPSS World language from Minuteman Software.

The linear project simulation tests were performed in line with the proposed method but disregarding the learning-forgetting effect, with the earliest possible times adopted as process start dates. Based on the results, a baseline contract period of $T_{d}=668$ days was agreed upon, with $60 \%$ probability of timely completion. The overall idle time for all work crews on all floors was 834 days. The tests were repeated with the learning-forgetting effect factored in. The probability of $T_{d}=594$ days was $60 \%$, with idle time equal to 624 days. Fig. 2 presents distribution functions, while fig. 3 contains total idle times for selected 
solutions adjusted for time buffers. The suboptimal solution features the following buffer lengths: $d_{12}=87, d_{23}=15, d_{34}=271, d_{45}=67$ days. The idle time was reduced to 136 days in all, which means 9.07 idle days for each floor, or 1.81 average idle time for each crew.

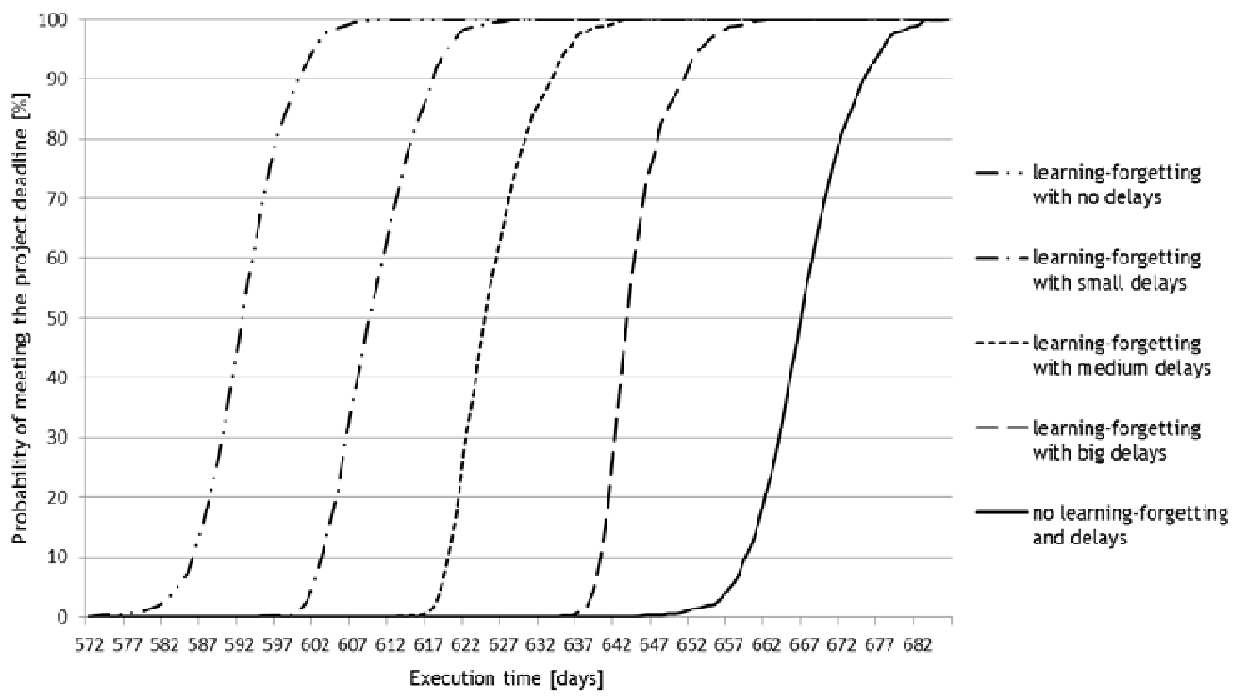

Fig. 2. Probability of meeting the project deadline.

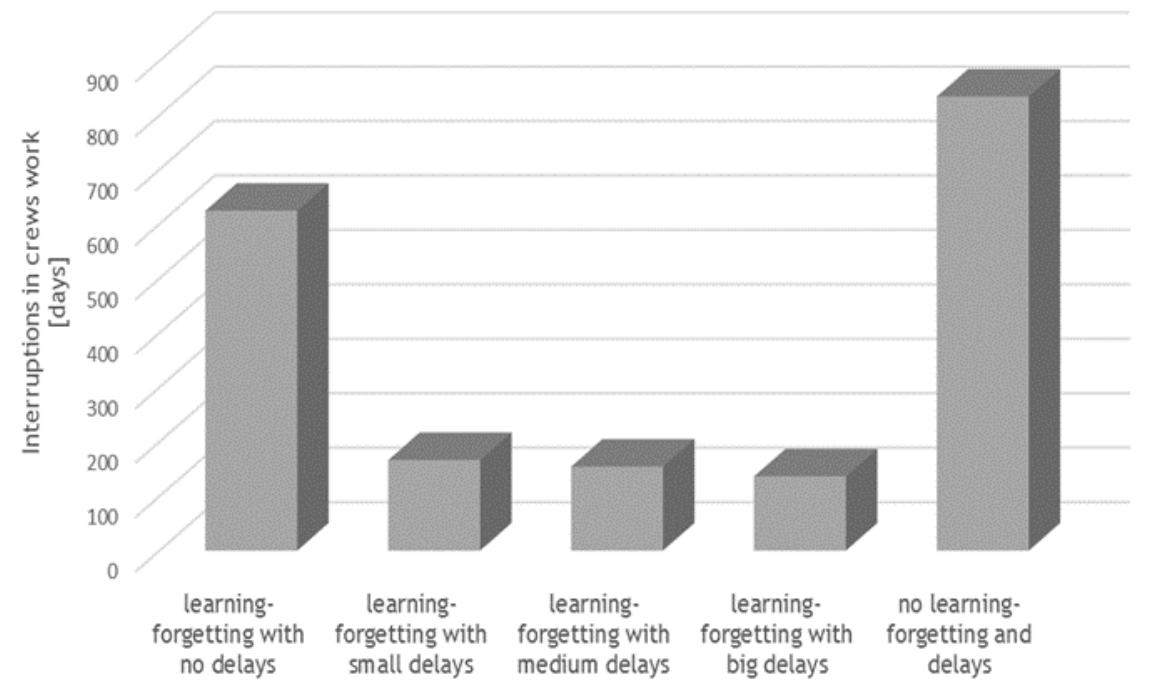

Fig. 3. Summed idle times in crews work.

\section{Conclusions}

Reference should be made to the learning-forgetting effect in scheduling linear projects such as high-rise buildings, roads and pipelines. In this type of projects it is possible to apply a division into units where the same of processes sequence is conducted. Learning effect allows the project lead time to be estimated with more precision as well as ensures a competitive edge at the bidding stage. Scheduling project work requires construction- 
specific risk factors to be considered in order to prevent the schedule from becoming outdated and, in particular, to anticipate finish date delays. Linear project schedules can be protected from disruptions allowing for delayed beginnings of successive processes (time buffers). It is also important to strive to eliminate idle periods which result when a preceding crew takes more time than planned to complete processes on a unit. Experience shows that delaying the work of crews carrying out repetitive processes contributes to reducing idle time as well as minimizing the forgetting effect, which leads to shorter lead times. At the same time, time buffers do not affect the baseline finish date. Research on the influence of experience and forgetting effect as well as time dispersion should be of interest to construction professionals and scholars alike, as it allows a more precise estimation of the duration of construction processes. Incorporating the Monte Carlo simulation, the proposed method allows linear construction project planning to take account of random conditions and learning-forgetting effect, while time buffers help to keep to the base line finish date at a given probability level, while also reducing idle time. The main problem in this kind of analyses is the need to have a high-quality inputs, eg data from completed projects.

The majority of construction projects, including linear projects, are affected by risk factors. The variety of processes, a large number of participants in the project as well as long implementation cycles make the construction project exposed to many external factors. If the project is very complicated and is carried out for a long time, the impact of risk factors increases due to problems related to the probability estimation of their occurrence and the assessment of their possible effects. The main limitations for the proposed method is the selection of a linear project with a few activities to minimize the impact of risk factors, as well as determining the optimal size of time buffers, which will not disturb the implementation cycles duration, thus shifting the project deadline.

Other of the primary limitations of the developed methodology in its present form is that it cannot handle intermittent activities. Also, the study focused on a project with unlimited access to resources. Nonetheless, the system can be adapted in the future for accommodating the intermittent activities, varying resource rates, limited resources, among other aspects.

This work was financially supported by Ministry of Science and Higher Education within the statutory research number $\mathrm{S} / 63 / 2018$.

\section{References}

1. D.W. Johnston, Linear scheduling method for highway construction, Journal of the Construction Division, 107(2), 247-261 (1981)

2. G. Lucko, Productivity scheduling method compared to linear and repetitive project scheduling methods, Journal of Construction Engineering and Management, 134(9), 711-720 (2008)

3. G. Lucko, Productivity Scheduling Method: Linear Schedule Analysis with Singularity Functions, Journal of Construction Engineering and Management, 135(4), 246-253 (2009)

4. J.J. O'Brien, VPM scheduling for high-rise buildings, Journal of the Construction Division, 101(4), 895-905 (1975)

5. R. Harris, P. Ioannou, Scheduling projects with repeating activities, Journal of Construction Engineering and Management, 124(4), 269-278, (1998) 
6. W.Y. Thabet, Y.J. Beliveau, HVLS: Horizontal and vertical logic scheduling for multistory projects, Journal of Construction Engineering and Management, 120(4), 875-892 (1994)

7. R. Flanagan, G. Norman, Risk management and construction, Blackwell Publishing. Oxford, UK, 1993

8. P. Zou, G. Zhang, J. Wang, Understanding the key risks in construction projects in China, International Journal of Project Management, 25, 601-614 (2007)

9. T.P. Wright, Factors affecting the cost of airplanes, Journal of the aeronautical sciences, 3(4), 122-128 (1936)

10. K.C. Lam, D. Lee, T. Hu, Understanding the effect of the learning-forgetting phenomenon to duration of projects construction, International Journal of Project Management, 19, 411-420 (2001)

11. S. Globerson, N. Levi, A. Shtub, Impact of breaks on forgetting when performing a repetitive task, IIE Transactions, 21(4), 376-381, (1989)

12. M.Y. Jaber, M. Bonney, Production breaks and the learning curve: the forgetting phenomenon, Applied Mathematical Modelling, 20(2), 162-169, (1996)

13. R. Pellegrino, N. Costantino, R. Pietrroforte, S. Sancilio, Construction of multi-storey concrete structures in Italy: patterns of productivity and learning curves. Construction Management and Economics, 30(2), 103-115 (2012)

14. M. Mályusz, A. Pém, Prediction of the learning curve in roof insulation, Automation in Construction, 36, 191-195 (2013)

15. J. Hinze, S. Olbina, Empirical analysis of the learning curve principle in prestressed concrete piles, Journal of Construction Engineering and Management, 135(5), 425-31 (2009)

16. A.M. Jarkas, Critical investigation into the applicability of the learning curve theory to rebar fixing labor productivity, Journal of Construction Engineering and Management, 136(12), 1279-1288 (2010)

17. A. Hassanein, O. Moselhi, Planning and scheduling highway construction, Journal of Construction Engineering and Management, 130(5), 638-646, (2004)

18. A. Panas, J.P. Pantouvakis, Simulation-based and statistical analysis of the learning effect in floating caisson construction operations, Journal of Construction Engineering and Management, 140(1), 04013033 (2013)

19. J. Lutz, D. Halpin, J. Wilson, Simulation of learning development in repetitive construction, Journal of Construction Engineering and Management, 120(4), 753-73 (1994) 